\title{
The Impact of COVID-19, Day-of-the-Week Effect, and Information Flows on Bitcoin's Return and Volatility
}

\author{
Ying Sing LIU' ${ }^{1}$ Liza LEE ${ }^{2}$
}

Received: August 01, 2020 Revised: September 28, 2020 Accepted: October 05, 2020

\begin{abstract}
Past literatures have not studied the impact of real-world events or information on the return and volatility of virtual currencies, particularly on the COVID-19 event, day-of-the-week effect, daily high-low price spreads and information flow rate. The study uses the ARMAGARCH model to capture Bitcoin's return and conditional volatility, and explores the impact of information flow rate on conditional volatility in the Bitcoin market based on the Mixture Distribution Hypothesis (Clark, 1973). There were 3,064 samples collected during the period from $1^{\text {st }}$ of January 2012 to $20^{\text {th }}$ April, 2020. Empirical results show that in the Bitcoin market, a daily high-low price spread has a significant inverse relationship for daily return, and information flow rate has a significant positive relationship for condition volatility. The study supports a significant negative relationship between information asymmetry and daily return, and there is a significant positive relationship between daily trading volume and condition volatility. When Bitcoin trades on Saturday \& Sunday, there is a significant reverse relationship for conditional volatility and there exists a day-of-the-week volatility effect. Under the impact of COVID-19 event, Bitcoin's condition volatility has increased significantly, indicating the risk of price changes. Finally, the Bitcoin's return has no impact on COVID-19 events and holidays (Saturday \& Sunday).
\end{abstract}

Keywords: Bitcoin, COVID-19 Event, Day-of-the-Week Effect, Daily High-Low Price Spread, Information Flow Rate

JEL Classification Code: C22, G10, G15

\section{Introduction}

Ever since the outbreak of the 2007-2008 global financial crisis, trust in traditional currency trading systems has been questioned, thus spurring the rapid development of cryptocurrencies based on a block chain of the Distributed Ledger Technology (DLT). Digital currency transactions utilize Bitcoin (BTC) as the main currency, have a distributed peer-to-peer payment system in the network, are between fiat and commodity currencies, have no intrinsic value, and are independent of any government or monetary authority (Baur, Hong \& Lee, 2018). Bitcoin's price has risen sharply since

${ }^{1}$ First Author and Corresponding Author. College of Humanities and Social Sciences, Chaoyang University of Technology, Taiwan [Postal Address: 168, Jifeng E. Road, Wufeng District, Taichung, 413330, Taiwan] Email: liuyingsing@yahoo.com.tw

${ }^{2}$ College of Humanities and Social Sciences, Chaoyang University of Technology, Taiwan. Email: Iylee@gm.cyut.edu.tw

(c) Copyright: The Author(s)

This is an Open Access article distributed under the terms of the Creative Commons Attribution Non-Commercial License (https://creativecommons.org/licenses/by-nc/4.0/) which permits unrestricted non-commercial use, distribution, and reproduction in any medium, provided the original work is properly cited.
2013, setting a record high trading price of 1 BTC to US\$ 1,9870.6 on December 17, 2017, giving global investors greater focus and attention.

Some scholars have a positive view of Bitcoin. Böhme, Christin, Edelman and Moore (2015) explain that Bitcoin is a kind of virtual currency that is used via online communication protocol and as an electronic payment system. This new payment system is not influenced by lawyers or regulators. Anyone can set up a Bitcoin account without paying fees, without undergoing any centralized review process, or without even providing a real name. Thus, in Bitcoin's rules, the payment system is more private, flexible, and less regulatory, potentially replacing existing payment and monetary systems. Carrick (2016) compares Bitcoin with currency values and volatility of emerging markets and finds that Bitcoin is well suited to complement emerging market currencies and presents a way to minimize Bitcoin's risk.

Some scholars have a negative view of Bitcoin. Gandala, Hamrick, Moore and Oberman (2018) believe that the use of Bitcoin can be severely influenced by security breaches and price volatility. Glaser, Zimmermann, Haferkorn, Webe and Siering (2014) state that users of digital currencies are 
primarily not interested in an alternative transaction system, but rather the primary purpose is to seek participation in an alternative investment vehicle. Bouoiyour (2015) notes that Bitcoin is classified as speculative foolery and offers no long-term promise. Yermack (2015) states that Bitcoin is more of a speculative investment than a currency, arguing that Bitcoin can only be applied in little consumer trading activities, and that there are still limits to it as functioning as a real currency such as a medium of exchange and value. At the same time, price volatility is much higher than real currencies, giving users larger short-term risk.

Bitcoin's value has little to do with real currencies and gold, faces risks of hacking and theft, and lacks a legal financial system for its protection and management. Böhme, Christin, Edelman and Moore (2015) suggest that there may be risks and regulatory issues with Bitcoin for the traditional financial system and the real economy. Corbet, Lucey and Yarovaya (2018) test the existence and dates of pricing bubbles in Bitcoin and Ethereum. It turns out that there are periods of clear bubble behavior, and the fact that Bitcoin is at the moment almost certainly in a bubble phase.

Bitcoin's pricing factors and market efficiency are the focus issues of many studies. Ciaian, Rajcaniova and Kancs (2016) examined Bitcoin's price formation and found that market forces, Bitcoin attractiveness for investors, and users have significant impact on Bitcoin's price, but with variations over time. Corbet, Larkin, Lucey, Meegan and Yarovaya (2020) analyze the relationship between news coverage and Bitcoin returns and show that an increase in good news (bad news) on unemployment and durable goods lead to a decline (upswing) in Bitcoin return, and that GDP and CPI-related news press releases have no significant relationship with Bitcoin's return. The results show that the developing cryptocurrency markets are further maturing through interactions with macroeconomic news. Alexander, Choi, Park and Sohn (2020) note that the prices of BitMEX derivatives at bid-ask spreads, interexchange spreads, and relative trading volumes are important factors in the discovery.

Some studies on the relationship between Bitcoin and traditional asset prices have not found a significant correlation between the two. Baur, Hong and Lee (2018) state that Bitcoin prices are not related to traditional asset prices (stocks, bonds, etc.), whether in normal times or periods of financial turmoil, and believe that Bitcoins are considered to be primarily used for speculative investment, rather than as an alternative currency and a medium of exchange. Using weekly data from 2010-2013, Brière, Oosterlinck and Szafarz (2015) found that Bitcoin has a very low correlation with other assets and has a particularly high average return and volatility. In addition, Huang (2019) looks at Bitcoin traders to assess the factors that affect the willingness to hold Bitcoin. The results show the following. (1) Most people know nothing about Bitcoin's values or risks. (2) If there are more transactions and speculative risks on Bitcoin, where less government intervention is expected, then government intervention does not affect the continued willingness of traders to hold onto their Bitcoins. (3) The higher the value of Bitcoin is, the more likely the trader fears the government intervention. (4) The government publicizes that Bitcoin's value and risk is distinguishable between gender, income, experience in digital currency use, and age of users in order to effectively regulate Bitcoin.

The results of many studies in the past have tended to suggest that Bitcoin is not market efficient. Urquhart (2016) explores the informational efficiency of Bitcoin and finds that returns are significantly inefficient, but supports that Bitcoin is being transformed from an inefficient market to an efficient market. Tiwari, Jana, Das and Roubaud (2018) found that the presence of Bitcoin market inefficiency existed from April 2013 to August of 2013 and from August 2016 to November of 2016. Shrestha (2019) states that Bitcoin returns are multifractal, thus supporting that the Bitcoin market is not efficient. In response to the question over Bitcoin's market efficiency, multifractality and inefficiency are thought to be the cause of the autocorrelated returns and extreme returns. Krückeberg and Scholz (2020) present that the Bitcoin market's inefficiency has increased over time. Urquhart (2017) found a significant evidence of price clustering in Bitcoin which shows a significant positive relationship in price-volume under price clustering. Mbanga (2019) shows that there are more Bitcoin price clusters on Fridays, but less on Mondays. Gandala, Hamrick, Moore and Oberman (2018) believe that Bitcoin currency exchange's suspicious trading activity is linked to a rise in the exchange rate, and that the Bitcoin market is vulnerable to manipulation in an unregulated situation.

Clark (1973) explores the relationship between daily return and trading volume based on the mixed distribution hypothesis (MDH); i.e., by assuming that the daily trading volume proxy measures the total amount of information arrival in the market that cannot be observed (Andersen, 1996; Lamoureux \& Lastrapes, 1990; Depken II, 2001; Bohl $\&$ Henke, 2003), and that changes in daily trading volume is a proxy variable for the information flow rate. Daily price volatility is the sum of each intra-day price change during the day, and daily volume is proportional to the number of information arrivals. There is a positive correlation between volatility and the information arrival rate. In addition, the bidask spreads are an important factor for the price discovery of BitMEX derivatives (Alexander, Choi, Park \& Sohn, 2020). Barclay and Smith (1988) use the form of relative bid-ask spread as a proxy variable for investors with information asymmetry in the trading market.

The impact of major events on the stock market has been a concern, and such issues have been explored (Tahir, 
Tahir, Syed, Ahmad, \& Ullah, 2020). Khan, Zhao, Zhang, Yang, Shah and Jahanger (2020) explored the impact of COVID-19 pandemic on the world's major stock markets. Alam, Alam and Chavali (2020) confirm that the impact of the lockdown caused by the COVID-19 to the stock market of India, and the results of the market reacted positively with the stock returns. The effect of COVID-19 events on the price behavior of virtual currencies has not been studied to discuss them. This study explores the impact of daily bidask price spreads and information flow rate on the return and volatility of the Bitcoin spot market. In past efficiency studies that have explored the Bitcoin market, less time has been spent on COVID-19 and for Saturday \& Sunday when analyzing the impact of Bitcoin's daily return and volatility. After the experiment brought through this study, the results clarify the effects of COVID-19 events and the day-of-theweek effects, and whether they are driven by information factors that significantly affect Bitcoin's price behavior.

\section{Research Methodologies}

\subsection{MDH}

Clark (1973) proposed the Mixture Distribution Hypothesis (MDH), which can be used to illustrate the relationship between price changes and daily trading volume (the amount of information flowing into the market). When Bitcoin does every transaction on intraday, a new equilibrium return $\mathrm{ER}_{i, t}$ is generated, which is, $i=1,2, \ldots, N_{t}$. If the daily return change is $\varepsilon_{t}$, the return change is caused by the sum total of each equilibrium return $E R_{i, t}$ on intraday. Therefore, daily return change $\varepsilon_{t}$ can be made in the form:

$$
\varepsilon_{t}=\sum_{i=1}^{N t} E R_{i, t}
$$

In the Eq. (1), $N_{t}$ is the number of equilibrium returns on intraday, where $N_{t}$ is the amount of information that is considered to have arrived in the market within trading day t. Assuming that $N_{t} \sim$ i.i.d $N\left(0, \sigma^{2}\right)$ and $\mathrm{N}_{t}$ is a mixed variable, the daily return change $\varepsilon_{t}$ should conform to the theoretical basis of $\mathrm{MDH}$. In the Eq. (1), the daily return change is generated by the subordinated stochastic process. If the amount of information flowing into the market is sufficient, then $\mathrm{N}_{t}$ is large enough. According to the Central Limit Theorem, conditional return changes can be expressed as:

$$
\varepsilon_{t} \mid N_{t} \sim N\left(0, \sigma^{2} \cdot N_{t}\right)
$$

Suppose the number of information flowing into the market is $N_{t}$, and $N_{t}$ subordinate the $\mathrm{AR}(\mathrm{p})$ model of autoregressive process:

$$
N_{t}=c_{0}+\sum_{i=1}^{p} \varnothing_{i} \cdot N_{t-1}+u_{t}
$$

In the Eq. (3), $\varnothing_{i}$ is the coefficient of the autoregressive item and $u_{t}$ is the error item. The conditional variance in the daily return that defines is as follows:

$$
\sigma_{\epsilon_{t} \mid N_{t}}^{2}=E\left(\epsilon_{t}^{2} \mid N_{t}\right)=\sigma^{2} \cdot N_{t}
$$

If the Eq. (3) is substituted to the Eq. (4), the conditional variance in the daily return is:

$$
\sigma_{\epsilon_{t} \mid N_{t}}^{2}=\sigma^{2} \cdot c_{0}+\sum_{i=1}^{p} \varnothing_{i} \cdot \sigma_{\epsilon_{t-i} \mid N_{t-i}}^{2}+\sigma^{2} \cdot u_{t}
$$

In the above, the Eq. (3) is inferable to the Eq. (5) and conforms to the GARCH pattern (Clark, 1973; Lamoureux \& Lastrapes, 1990). As the rate at which information arrives to the market changes over time, the volatility in the market also changes with the rate at which information arrives.

\subsection{Empirical Model}

Corwin and Schultz (2012) support the use of daily high-low prices to estimate bid-ask spreads, arguing that this approach can be applied to a variety of research areas and is better than other low-frequency estimators. After this study refers to the form of relative bid-ask spread proposed by Barclay and Smith (1988), the calculation formula for defining the daily high-low price spread (Paule-Vianez, Prado-Román \& Gómez-Martínez, 2020) is:

$$
\begin{aligned}
\text { Spread }_{t} & =\frac{\left|P_{t}^{\text {high }}-P_{t}^{\text {low }}\right|}{\text { Mid }_{t}} \\
\text { Mid }_{t} & =\frac{\left|P_{t}^{\text {high }}+P_{t}^{\text {low }}\right|}{2}
\end{aligned}
$$

In Eq. (6) and Eq. (7), Spread represents the high-low price spread on the $t^{\text {th }}$ day; $P_{t}^{\text {high }}$ is a high price on the $t^{\text {th }}$ day; $P_{t}^{\text {low }}$ is a low price on the $t^{\text {th }}$ day; and Mid $t_{t}$ is the mid-point of the trading price on the $t^{\text {th }}$ day.

Bariviera (2017) confirms that Bitcoin's daily return exhibits persistent behavior, and so this present study considers the lagged term of the daily return as an explanatory variable, which is analyzed using the Autoregressive Moving Average (ARMA) model. Before the ARMA model can be established, the study series must be identified as stationary, and the unit root test of the Phillips-Perron test proposed by Phillips and Perron (1988) is used. In addition, there is a conditional variance $h_{t}$ in the error term $\varepsilon_{t}$, and the condition of the information set given $\Omega_{t-1}$ is the previous period, where $\varepsilon_{t} \mid \Omega_{t-1} \sim N\left(0, h_{t}\right)$. The error term $\varepsilon_{t}$ is based on the heteroskedasticity ARCH effect test to infer the existence of heteroscedasticity. When the square error is heterogeneous, the GARCH model is used as a conditional variance equation 
(Depken II, 2001; Dyhrberg, 2016). Bollerslev, Chou and Kroner (1992) believe that the GARCH $(1,1)$ model can capture the volatility characteristics of most time series.

This study is analyzed using the ARMA (p, q) and GARCH $(1,1)$ models, and the effect of replacing the information flow rate (change of trading volume) into the GARCH model is likely to reduce persistence or even make it completely disappear (Gallo \& Pacini, 2000; Bohl \& Henke, 2003). In addition, the daily high-low price spread can be used as daily uncertainty or the partly due to information asymmetry, further testing the relationship between highlow price spread and daily return. The model for this study is therefore as follows:

$$
\begin{aligned}
R_{t}= & a_{0}+\sum_{i=1}^{p} a_{i} \cdot R_{t-i}+\sum_{j=1}^{q} b_{j} \cdot \varepsilon_{t-j} \\
& +c \cdot \text { Spread }_{t}+d \cdot H D_{t}+e \cdot C D_{t}+\varepsilon_{t}
\end{aligned}
$$

where $\varepsilon_{t} \mid \Omega_{t-1} \sim N\left(0, h_{t}\right)$

$$
h_{t}=\omega+\alpha \cdot \varepsilon_{t-1}^{2}+\beta \cdot h_{t-1}+\gamma \cdot I F R_{t}+\delta \cdot H D_{t}+\theta \cdot C D_{t}
$$

In Eq. (8) and Eq. (9), all estimation parameters are the conditional density of all predicted errors calculated by using the maximum likelihood method. The log-likelihood function calculates the conditional density of all prediction errors. When the error term is assumed to be a standard normal distribution, the available log-likelihood function is:

$$
l=\sum_{i=1}^{N} \frac{1}{2}\left[-\operatorname{Ln}(2 \pi)-\operatorname{Ln}\left(h_{t}\right)-\frac{\varepsilon_{t}^{2}}{h_{t}}\right]
$$

In Eq. (8) and Eq. (9), $R_{t}$ is the daily return on the $t^{\text {th }}$ day; $R_{t-i}$ and $\varepsilon_{t-j}$ are daily return and error terms on the lagged $i^{\text {th }}$ day and $j^{\text {th }}$ day; $h_{t}$ is a condition variance equation for daily return; $\alpha_{0}$ and $\omega$ are intercept items; Spread is the highlow price spread on the $t^{\text {th }}$ day; $I F R_{t}$ is the daily information arrival rate on the $t^{\text {th }}$ day; $H D_{t}$ is a dummy variable for holiday, and assuming that the $t^{\text {th }}$ day is Saturday or Sunday, then $H D_{t}$ equals 1 and otherwise 0 (This way of defining dummy variable is by referencing Liu and Chen (2011)); and $C D_{t}$ is a dummy variable for COVID-19, and assuming that the $t^{\text {th }}$ day during after the announcement of Wuhan, China being put on lockdown to contain the spread of the virus on January 23, 2020, the given $C D_{t}$ equals 1 and otherwise 0 .

\subsection{Null Hypothesis}

This study examines seven null hypotheses for the estimation parameters in Eq. (8) and Eq. (9) to infer the relationship between the daily high-low price spread and return. For the impact of the information arrival rate for conditional volatility, there are the day-of-the-week effects and the COVID-19 effect on the daily return or conditional volatility. The seven null hypotheses are as follows:

$\boldsymbol{H}_{10}: \boldsymbol{c} \geq \mathbf{0}$. If $C<0$ supports the significant reverse relationship between the daily high-low price spread and the daily return. This result is implicit in the Bitcoin market, where a significant difference in information sets between the buyer and seller, will result in a significant decrease in Bitcoin's daily return if there is greater information asymmetry.

$\boldsymbol{H}_{\mathbf{2 0}}: \boldsymbol{d}=\mathbf{0}$. If $\boldsymbol{H}_{20}$ is rejected, then accept return has a significant day-of-the-week effect.

$\boldsymbol{H}_{30}: \boldsymbol{e}=\mathbf{0}$. If $\boldsymbol{H}_{30}$ is rejected, then accept an effect on Bitcoin's return during the COVID-19.

$\boldsymbol{H}_{40}: \gamma \leq \mathbf{0}$. If $\boldsymbol{H}_{40}$ is rejected, then $\gamma>0$ indicates a significant positive relationship with the information arrival rate and volatility.

$\boldsymbol{H}_{\mathbf{5 0}}: \delta=\mathbf{0}$. If $\boldsymbol{H}_{\mathbf{5 0}}$ is rejected, then accept Bitcoin is being traded on a holiday and has a correlation for Bitcoin's conditional volatility, and there is a day-of-the-week effect.

$\boldsymbol{H}_{60}: \theta=\mathbf{0}$. If $\boldsymbol{H}_{60}$ is rejected, then accept a correlation for Bitcoin's conditional volatility during the COVID-19, and therefore the COVID-19 effect is supported.

$\boldsymbol{H}_{70}: \alpha \leq \mathbf{0}$ and $\beta \leq \mathbf{0}$. If $\boldsymbol{H}_{70}$ is rejected, then accept $\alpha>0$ and $\beta>0$, and these results support the existence of the GARCH effect and ARCH effect.

\section{Data and Empirical Results}

This study collected Bitcoin historical trading data from the investing.com website (Source: https://www.investing. com/crypto/bitcoin /historical-data) for analysis. The data include Bitcoin's daily closing price (Unit: USD; 1 BTC can be exchanged for how many US Dollars), high price, low price, and trading volume (Unit: K BTC is a 1,000 BTC). There were 3,064 trading days from January 1, 2012 to April 20, 2020.

Figure 1 presents the statistical charts of the study variables, which contain time series such as daily closing price, daily trading volume, return (change of closing price), information flow rate, and daily high-low price spread. Chart 1 is a line chart of Bitcoin's daily closing price, and Chart 2 is a bar chart of Bitcoin's daily trading volume, which mainly allows us to observe the long-term changes in Bitcoin's price movements and trading volume. In Chart 1 and Chart 2, we see a long-term trend of rapid price increases in the Bitcoin market during 2017 after exceeding US\$1000 per 1 BTC (2017/2/2: closing price: US\$1004 per 1 BTC), and hitting a record high trading price of US $\$ 19,870.6$ per 1 BTC on December 17, 2017. For daily trading volume, more than one million Bitcoins are traded in the market every day after mid-2018, showing the market to have hot trading volume. 

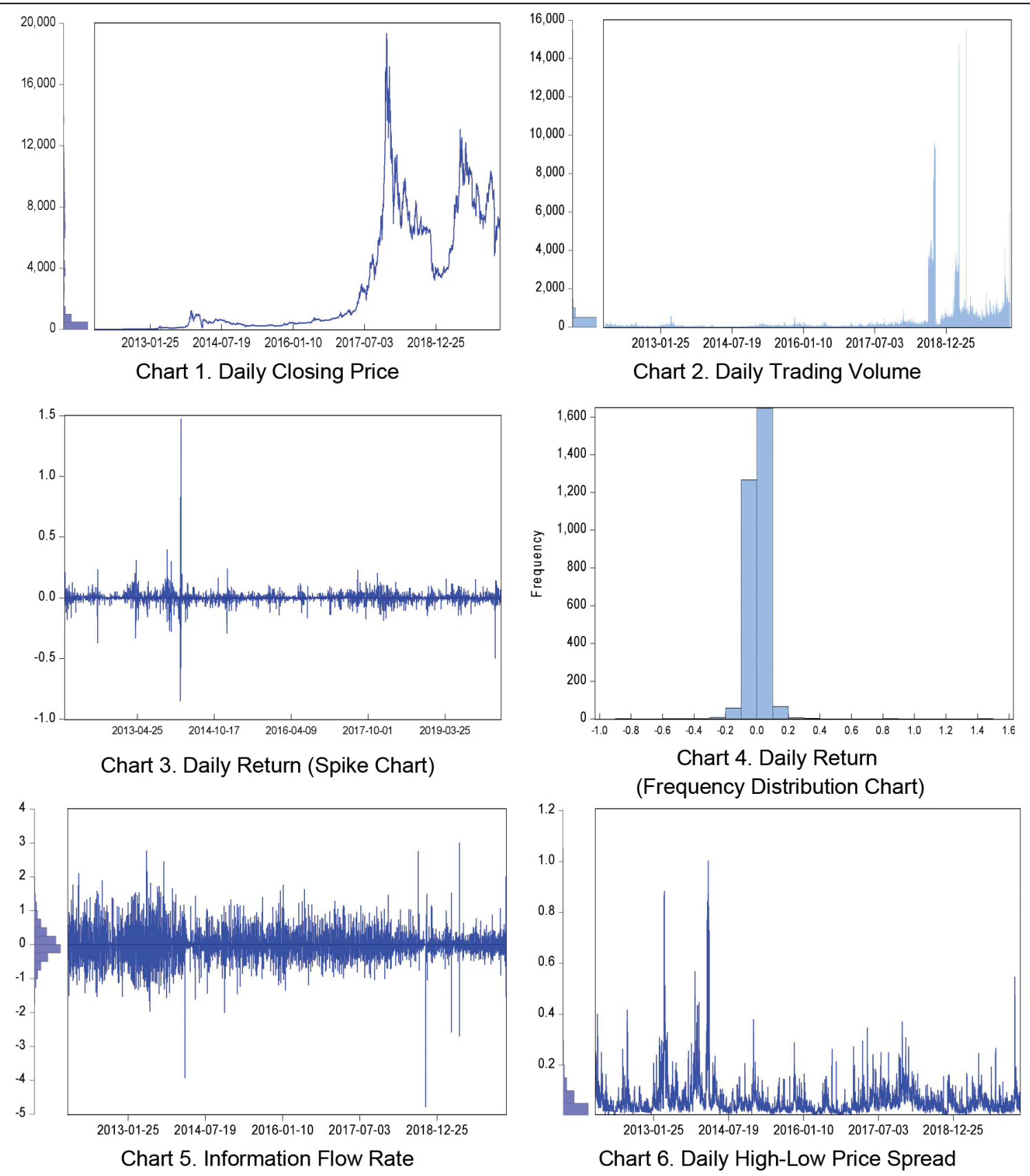

Figure 1: Statistical Diagram of Study Variables $(O b s .=3064)$

Chart 3 and Chart 4 are the spike chart and frequency distribution chart of Bitcoin's daily return, respectively. In Chart 3, large concentrations of price volatility (spike) occurred between the second quarter of 2013 and the first quarter of 2014, the beginning of 2017 and the second quarter of 2018, and the first quarter of 2020 to the end of the statistical period. A larger price volatility is found to be concentrated during these periods. In Chart 4 , we see that the daily return is roughly between the -0.1 and 0.1 range $( \pm 10 \%)$, with the largest frequency ranging from 0.0 to 0.1 . Chart 5 is a spike chart of the information flow rate. On the left side of the Chart 5 is the frequency distribution chart, and 
we find that the information flow rate falls roughly between the -1.0 and $1.0( \pm 100 \%)$ range. During the trading period prior to the beginning of 2014, the information flow rate in the Bitcoin market is more volatile and varies significantly. Chart 6 is a spike chart for the daily high-low price spread, where we find it to be similar to Chart 5. The high-low price spreads are generally higher before the beginning of 2014 than after the beginning of 2014.

In the beginning of the 2020 outbreak of COVID-19 during the early stages, we find that the daily high-low price spread has suddenly increased, showing that COVID-19 generates larger uncertainty for Bitcoin's price. On the left side of Chart 5 is a frequency distribution chart. Here, we see that the highlow price spread is roughly in the range of less than 0.2 .

Table 1 is the result of the descriptive statistics and the unit root tests, with 3,064 trading days from January 1, 2012 to April 20, 2020. We use the Phillips-Perron tests to test whether these time series are stationary, taking into the equation the intercept and time trend items.

In Table 1, the means (Std. Dev.) of daily closing price, daily trading volume $(\mathrm{K})$, daily return, information flow rate, and daily high-low price spread equal US\$2730.31 (3692.4630), 311.66 K BTC (938.0579), 0.0024 (0.0588), 0.0007 (0.5161), and $0.0613(0.0709)$, respectively. The means of the daily return and information flow rate (change of daily trading volume) are positive values, indicating that investment in Bitcoin is profitable over the long term, and the market is gradually growing in trading size. In the coefficient of skewness, we find that only the coefficient of skewness of the information flow rate is less than zero $(-0.0417)$ and is tail outward to the left side of the distribution. The other four series have a coefficient of skewness greater than zero and a tail outward to the right side of the distribution. We further see that the Kurtosis of the five series are greater than 3, presenting leptokurtic for these series. At the 5\% level, the Jarque-Bera tests results of the five series are significant, indicating that all series show a non-normal distribution. Finally, in the results of the Phillips-Perron tests, we find that the estimated p-value less than 0.05 from the daily return, the information flow rate, and the daily high-low price spread, rendering significant support for the three time series being stationary.

Table 2 lists the results of the estimated parameters on the study model, by using the maximum likelihood estimation of ARMA on the parameters. In the process of building the model, the return equation, after adding the independent variable of the daily high-low price spread, uses the $Q(15)$ value to diagnose the autocorrelation of the error term. This is more appropriate for determining the ARMA $(1,1)$ model by the SBC value being the smallest, where the order of AR is $p=1$ and the order of MA is $q=1$. Second, we use the Heteroskedasticity ARCH effect test to infer whether there is heterogeneity on the square error of the $\operatorname{ARMA}(1,1)$ model. When the square error of the ARMA $(1,1)$ model is heterogeneous, it significantly captures heterogeneity with the GARCH $(1,1)$ model. Finally, in Table 2, from the diagnostic checking and goodness-of-fit screening process on the model, we note that the smallest SBC value is -3.5880 , where $p=1$ and $q=1$. Here, Q (15) is 15.797 , and the $p$-value is 0.260 , thus supporting that the error term does not exhibit autocorrelation. In the Heteroskasticity ARCH effect test, the F-statistic/p-value is $0.1906 / 0.6624$, and there is no heterogeneity.

Table 1: Descriptive Statistics and the Unit Root Tests (Obs. =3064)

\begin{tabular}{|l|c|c|c|c|c|}
\hline $\begin{array}{l}\text { Variable } \\
\text { Estimator }\end{array}$ & Close price & $\begin{array}{c}\text { Daily trading } \\
\text { volume (K) }\end{array}$ & Daily return & $\begin{array}{c}\text { Information flow } \\
\text { rate }\end{array}$ & $\begin{array}{c}\text { Daily high-low } \\
\text { price spread }\end{array}$ \\
\hline Mean & 2730.31 & 311.66 & 0.0024 & 0.0007 & 0.0613 \\
\hline Median & 592.95 & 65.76 & 0.0010 & -0.0212 & 0.0414 \\
\hline Maximum & 19345.50 & 15430.00 & 1.4742 & 3.0065 & 1.0013 \\
\hline Minimum & 4.20 & 0.40 & -0.8488 & 0.7802 & 0.5161 \\
\hline Std. Dev. & 3692.46 & 938.06 & 0.0588 & -0.0417 & 5.0709 \\
\hline Skewness & 1.3637 & 8.2493 & 4.3600 & 8.5749 & 47.9353 \\
\hline Kurtosis & 3.9939 & 93.0659 & 164.7920 & $3968.73^{* * *}$ & $272057^{* * *}$ \\
\hline Jarque-Bera & $1075.82^{* * *}$ & $1070366^{* * *}$ & $3351595^{* * *}$ & $0.0001^{* * *}$ & $0.0000^{* * *}$ \\
\hline $\begin{array}{l}\text { Phillips-Perron } \\
\text { test (p-value) }\end{array}$ & 0.1881 & $0.0001^{* * *}$ & $0.0000^{* * *}$ & 2870 \\
\hline
\end{tabular}

Note: ${ }^{* * *},{ }^{* *}$ and ${ }^{*}$ represent significance at the $1 \%, 5 \%$ and $10 \%$ levels, respectively. 
Table 2 shows that the coefficient of AR(1) is $\alpha_{1}=0.9735$ ( $\mathrm{p}$-value $=0.0000<0.01)$ is significantly positive, indicating that the relationship between the return of the previous day and the return of the day is significantly positive in the Bitcoin market; i.e., the return of the previous day is positive (up), and there is a positive effect on the return of the day. The coefficient of MA(1) is $b_{1}=-0.9489$ (p-value $=0.0000<$ 0.01 ), which is significantly negative, indicates that Bitcoin's error term on the previous day is inversely related to the return of the day. Null hypothesis I $\left(\boldsymbol{H}_{\mathbf{1 0}}\right)$ testing $c \geq 0$, when $c<0$ shows a significant reverse relationship on the daily high-low spread and daily return. The estimated parameter of the daily high-low price spread equally -0.0878 (p-value $=0.0000<$ 0.01 ), rendering it significantly less than zero and showing a significant reverse relationship on the daily high-low price spread (Spread) and daily return. Assuming that the daily high-low price spread is partly the result of a discrepancy in the trader's information, the degree of information difference has a significant reverse effect on the daily return. This result proves that Bitcoin traders have a negative effect on Bitcoin's daily return when there is information asymmetry.

Trading in the Bitcoin market is different from real currencies, especially when financial markets are closed, which is a custom on Saturdays and Sundays. However, Bitcoin markets continue to trade on Saturday \& Sunday. The null hypothesis II $\left(\boldsymbol{H}_{\mathbf{2 0}}\right)$ tests $d=0$, and when the estimate parameter $d=-0.0007$ (p-value $=0.6802>0.05)$, it is impossible to reject the null hypothesis II $\left(\boldsymbol{H}_{\mathbf{2 0}}\right)$, showing that the Bitcoin trading market during a holiday period does not affect the daily return of the average level. For the null hypothesis III $\left(\boldsymbol{H}_{\mathbf{3 0}}\right)$ of testing $e=0$, when the estimation parameter $e=-0.0056$ ( $\mathrm{p}$-value $=$ $0.6667>0.05)$, it is impossible to reject this null hypothesis, indicating that the period during which COVID-19 occurs does not affect Bitcoin's daily average return.

Table 2: The Results of Estimated Parameters on the Study Model (Obs. =3064)

\begin{tabular}{|c|c|c|c|c|}
\hline Variable & Coefficient & Std. Dev. & z-statistic & p-value \\
\hline \multicolumn{5}{|l|}{ Mean Equation } \\
\hline Constant & 0.0039 & 0.0028 & 1.3845 & 0.1662 \\
\hline$R_{t-1}$ & $0.9735^{\star \star *}$ & 0.0144 & 67.7094 & 0.0000 \\
\hline$\varepsilon_{t-1}$ & $-0.9489^{* * *}$ & 0.0200 & -47.5361 & 0.0000 \\
\hline Spread $_{t}$ & $-0.0878^{* * *}$ & 0.0098 & -8.9986 & 0.0000 \\
\hline$H D_{t}$ & -0.0007 & 0.0018 & -0.4122 & 0.6802 \\
\hline$C D_{t}$ & -0.0056 & 0.0129 & -0.4307 & 0.6667 \\
\hline \multicolumn{5}{|l|}{ Variance Equation } \\
\hline Constant & $0.0012^{* * *}$ & 0.0001 & 22.8506 & 0.0000 \\
\hline$\varepsilon_{t-1}^{2}$ & $0.2859^{* * *}$ & 0.0150 & 19.0681 & 0.0000 \\
\hline$h_{t-1}$ & $0.4416^{\star \star \star}$ & 0.0152 & 29.0379 & 0.0000 \\
\hline$I F R_{t}$ & $0.0003^{* \star *}$ & 0.0001 & 48.4475 & 0.0000 \\
\hline$H D_{t}$ & $-0.0011^{* * *}$ & 0.0000 & -24.5154 & 0.0000 \\
\hline$C D_{t}$ & $0.0004^{* * *}$ & 0.0001 & 3.6006 & 0.0003 \\
\hline R-squared & 0.0126 & Schwarz Criterion & \multicolumn{2}{|c|}{-3.5880} \\
\hline Adjusted R-squared & 0.0110 & Durbin-Watson Stat. & \multicolumn{2}{|c|}{2.0312} \\
\hline Log likelihood & 5618.697 & $\mathrm{Q}(15) / p$-value & \multicolumn{2}{|c|}{$15.797 / 0.260$} \\
\hline \multicolumn{2}{|c|}{$\begin{array}{l}\text { Heteroskedasticity Test: ARCH F-statistic/ } \\
\text { p-value }\end{array}$} & \multicolumn{3}{|c|}{$0.1906 / 0.6624$} \\
\hline
\end{tabular}

Note: ${ }^{* * *},{ }^{* *}$ and ${ }^{*}$ represent significance at the $1 \%, 5 \%$ and $10 \%$ levels, respectively. 
In the conditional variance equation, the null hypothesis IV $\left(\boldsymbol{H}_{40}\right)$ tests $\gamma \leq 0$, and when the estimate parameter $\gamma=$ 0.0003 (p-value $=0.0000<0.01)$ is significantly greater than zero, it rejects $\mathrm{H}_{40}$ and accepts a significant positive relationship on the information flow rate $\left(I F R_{t}\right)$ and conditional volatility, showing that a larger information flow rate in the Bitcoin market will be significantly larger for Bitcoin's price volatility. The null hypothesis $\mathrm{V}\left(\boldsymbol{H}_{50}\right)$ tests $\delta=0$, and when the estimated parameter $\delta=-0.0011$ (p-value $=0.0000<0.01)$ is significantly less than zero, it rejects $\boldsymbol{H}_{\mathbf{5 0}}$ and accepts $\delta<0$, showing that the Bitcoin trading market on Saturday \& Sunday will significantly reduce volatility, supporting the existence of a day-of-the-week effect. The results confirm in the real world that, as humans work and leisure activities change, there will be a significant degree of change in Bitcoin's price on conditional volatility. The null hypothesis VI $\left(\boldsymbol{H}_{60}\right)$ testing $\theta=0$, exploring the impact on Bitcoin's volatility during the COVID-19 period. When the estimated parameter $\theta=0.0004$ ( $\mathrm{p}$-value $=0.0003<0.01$ ), it show that as humans in the real world feel threatened by more uncertainty (risk) during the COVID-19 period, the uncertainty (risk) spreads to the virtual world, and so Bitcoin's price volatility significantly increases. It is confirmed that the COVID-19 event significantly affects Bitcoin's price volatility, supporting the presence of the COVID-19 effect.

The null hypothesis VII $\left(\boldsymbol{H}_{70}\right)$ tests $\alpha \leq 0$ and $\beta \leq 0$, and when the estimated parameter $\alpha=0.2859$ (p-value $=$ $0.0000<0.01)$ and $\beta=0.4416$ ( $\mathrm{p}$-value $=0.0000<0.01$ ) present significant results, there is a GARCH effect and ARCH effect. Thus, Bitcoin's conditional volatility is similar to the financial asset price volatility, and there is a clustering and persistence phenomenon. In addition, when $\alpha+\beta=0.2859+0.4416=0.7275$ (when $\alpha+\beta$ is close to 1 without the information flow rate), it shows that the effect on volatility is to reduce the persistence of the property with the information flow rate (change of trading volume) is added to the GARCH model. This result shows that only part of Bitcoin's volatility can be explained by the information flow rate, unlike Lamoureux and Lastrapes (1990) who validate results in the stock market that make the sustainability of volatility disappear. This study finds in the Bitcoin market that the ability of the information flow rate to affect volatility is significant but less powerful than the effect of the stock market.

\section{Conclusions}

This paper focuses on the COVID-19 events, the day-ofthe-week effect, and trading information on Bitcoin's daily return and volatility. From the empirical results, we find that Bitcoin's daily return is an auto-regressive and movingaverage process, and there is heteroscedastic variance. The daily high-low price spread in the Bitcoin market has a reverse relationship with the daily return. There is a significantly positive relationship between the information flow rate and volatility, and when the information flow rate is added to the conditional variance equation, we see that the impact on volatility is less effective than that of the stock market. The COVID-19 and holiday effects exist in Bitcoin's volatility, but not in the daily return. The tests of this study support the impact of COVID-19 events, the day-of-theweek effects (Saturday \& Sunday), and trading information on Bitcoin's price behavior.

\section{References}

Alam, M. N., Alam, M. S., \& Chavali, K. (2020). Stock market response during COVID-19 lockdown period in India: An event study. Journal of Asian Finance, Economics and Business, 7(7), 131-137. https://doi.org/10.13106/jafeb.2020.vol7.no7.131

Alexander, C., Choi, J., Park, H., \& Sohn, S. (2020). BitMEX bitcoin derivatives: Price discovery, informational efficiency, and hedging effectiveness. Journal of Futures Markets, 40(1), 23-43. https://doi.org/10.1002/fut.22050

Andersen, T. (1996). Return volatility and trading volume: An information flow interpretation of stochastic volatility. Journal of Finance, 51(1), 169-204. https://doi. org/10.1111/j.1540-6261.1996.tb05206.x

Barclay, M., \& Smith, C. W. J. (1988). Corporate payout policy: Cash dividends versus open-market repurchases. Journal of Financial Economics, 22(1), 61-82. https://doi. org/10.1016/0304-405X (88)90022-0

Bariviera, A. F. (2017). The inefficiency of Bitcoin revisited: A dynamic approach. Economics Letters, 161, 1-4. https://doi. org/10.1016/j.econlet.2017.09.013

Baur, D. G., Hong, K. H., \& Lee, A. D. (2018). Bitcoin: Medium of exchange or speculative assets?. Journal of International Financial Markets, Institutions and Money, 54, 177-189. https://doi.org/10.1016/j.intfin.2017.12.004

Bohl, M. T., \& Henke, H. (2003). Trading volume and stock market volatility: The Polish case. International Review of Financial Analysis, 12(5), 513-525. https://doi.org/10.1016/S1057-5219(03)00066-8

Böhme, R., Christin, N., Edelman, B., \& Moore, T. (2015). Bitcoin: Economics, Technology, and Governance. Journal of Economic Perspectives, 29(2), 213-238.

Bollerslev, T., Chou, R. Y., \& Kroner, K. F. (1992). ARCH modeling in Finances a review of the theory and empirical evidence. Journal of Econometrics, 52(1-2), 5-59. https://doi. org/10.1016/0304-4076(92)90064-X

Bouoiyour, J. (2015). What Does Bitcoin Look Like?. Annals of Economics and Finance, 16(2), 449-492. http://aeconf.com/ Articles/Nov2015/aef160211.pdf

Brière, M., Oosterlinck, K., \& Szafarz, A. (2015). Virtual currency, tangible return: Portfolio diversification with bitcoin. Journal of Asset Management, 16, 365-373. https://doi.org/10.1057/ jam.2015.5 
Carrick, J. (2016). Bitcoin as a complement to emerging market currencies. Emerging Markets Finance and Trade, 52(10), 2321-2334. https://doi.org/10.1080/1540496X.2016.1193002

Ciaian, P., Rajcaniova, M., \& Kancs, D. (2016). The economics of BitCoin price formation. Applied Economics, 48(19), 17991815. https://doi.org/10.1080/00036846.2015.1109038

Clark, P. K. (1973). A subordinated stochastic process model with finite variance for speculative prices. Econometrica, 41(1), 135156. https://doi.org/0012-9682(197301)41:1<135:ASSPMW $>2.0 . \mathrm{CO} ; 2-\mathrm{G}$

Corbet, S., Larkin, C., Lucey, B. M., Meegan, A., \& Yarovaya, L. (2020). The impact of macroeconomic news on Bitcoin returns. European Journal of Finance, 26(14), 1396-1416. https://doi. org/10.1080/1351847X .2020.1737168

Corbet, S., Lucey, B., \& Yarovaya, L. (2018). Datestamping the Bitcoin and Ethereum bubbles. Finance Research Letters, 26, 81-88. https://doi.org/10.1016/j.frl.2017.12.006

Corwin, S. A., \& Schultz, P. (2012). A simple way to estimate bid-ask spreads from daily high and low prices. Journal of Finance, 67(2), 719-760. https://doi.org/10.1111/j.15406261.2012.01729.x

Depken II, C. A. (2001). Good news, bad news and GARCH effects in stock return data. Journal of Applied Economics, 4(2), 313327. https://doi.org/10.1080/15140326.2001.12040567

Dyhrberg, A. H. (2016). Bitcoin, gold and the dollar: A GARCH volatility analysis. Finance Research Letters, 16, 85-92. https:// doi.org/10.1016/j.frl.2015.10.008

Gallo, G. M., \& Pacini, B. (2000). The effects of trading activity on market volatility. European Journal of Finance, 6(2), 163-175. https://doi.org/10.1080/13518470050020824

Gandala, N., Hamrick, J. T., Moore, T., \& Oberman, T. (2018). Price manipulation in the Bitcoin ecosystem. Journal of Monetary Economics, 95, 86-96. https://doi.org/10.1016 /j.jmoneco.2017.12.004

Glaser, F., Zimmermann, K., Haferkorn, M., Webe, M. C., \& Siering, M. (2014). Bitcoin-Asset or currency? Revealing users' hidden intentions. In: 22th European Conference on Information Systems (pp. 1-14). Tel Aviv, Israel, June 9-11. https://ssrn.com/abstract $=2425247$

Huang, W. (2019). The impact on people's holding intention of bitcoin by their perceived risk and value. Economic ResearchEkonomska Istraživanj, 32(1), 3570-3585. https://doi. org/10.1080/ 1331677X .2019.1667257

Khan,K., Zhao, H., Zhang, H., Yang, H., Shah, M. H., \& Jahanger, A. (2020). The impact of COVID-19 pandemic on stock markets: An empirical analysis of world major stock indices. Journal of Asian Finance, Economics and Business, 7(7), 463474. https://doi.org/10.13106/jafeb.2020. vol7.no7.463

Krückeberg, S., \& Scholz, P. (2020). Decentralized efficiency? Arbitrage in Bitcoin markets. Financial Analysts Journal, 76(3), 135-152. https://doi.org/10.1080/001519 8X.2020.1733902

Lamoureux, C., \& Lastrapes, W. (1990). Heteroscedasticity in stock return data: Volume versus GARCH effects. Journal of Finance, 45(1), 221-229. https://doi.org/10.1111/j.1540-6261.1990. tb05088.x

Liu, Y. S., \& Chen, Y. C. (2011). The day-of-the-week volatility effect in Taiwan's stock index futures market is puzzle. Journal of Interdisciplinary Mathematics, 14(6), 619-625. https://doi. org/10.1080/09720502. 2011.10700778

Mbanga, C. L. (2019). The day-of-the-week pattern of price clustering in Bitcoin. Applied Economics Letters, 26(10), 807811. https://doi.org/10.1080/13504851.2018.1497844

Phillips, P. C. B., \& Perron, P. (1988). Testing for a unit root in time series regression. Biometrika, 75(2), 335-346. https://doi. org/10.1093/biomet/75.2.335

Paule-Vianez, J., Prado-Román, C., \& Gómez-Martínez, R. (2020). Economic policy uncertainty and Bitcoin. Is Bitcoin a safehaven asset?. European Journal of Management and Business Economics, https://www.emerald.com/insight/2444-8494.htm

Shrestha, K. (2019). Multifractal detrended fluctuation analysis of return on Bitcoin. International Review of Finance, https://doi. org/10.1111/irfi.12256

Tahir, S. H., Tahir, F., Syed, N., Ahmad, G., \& Ullah, M. R. (2020). Stock market response to terrorist attacks: An event study approach. Journal of Asian Finance, Economics and Business, 7(9), 31-37. https://doi.org/10.13106/jafeb.2020.vol7.no9.031

Tiwari, A. K., Jana, R. K., Das, D., \& Roubaud, D. (2018). Informational efficiency of Bitcoin: An extension. Economics Letters, 163, 106-109. https://doi.org/10.1016/j. econlet.2017.12.006

Urquhart, A. (2016). The inefficiency of Bitcoin. Economics Letters, 148, 80-82. https://doi.org/10.1016/j.econlet.2016.09.019

Urquhart, A. (2017). Price clustering in Bitcoin. Economics Letters, 159, 145-148. https://doi.org/10.1016/ j.econlet.2017.07.035

Yermack, D. (2015). Chapter 2 - Is Bitcoin a real currency? An economic appraisal. In: D. Lee, \& K. Chuen (Eds.), Handbook of Digital Currency: Bitcoin, innovation, Financial Instruments, and Big Data (pp. 31-43), London, UK: Academic Press. https://doi.org/10.1016/B978-0-12-802117-0.00002-3 